\title{
El gabinete de prensa ante el nuevo ecosistema informativo digital
}

\author{
Rosa de Bustos \\ Periodista, responsable de Prensa de la UNE
}

Los gabinetes de prensa se han visto obligados a evolucionar en los últimos años como consecuencia de las sucesivas transformaciones de los medios de comunicación tradicionales - prensa, radio, televisión- y, más recientemente, de Internet. Unos cambios que, sin embargo y a mi modo de ver, no han alterado -hasta ahora- los esquemas de trabajo sobre los que se ha venido asentado la tarea informativa de las oficinas de comunicación. Es cierto que han ido cambiando las herramientas, formatos, soportes y rutinas, pero todas estas transformaciones se han desarrollado dentro del paradigma que ha regido esta actividad profesional en el último cuarto de siglo: los gabinetes de prensa elaboraban información (texto, imagen, sonido) y la difundían a los medios de comunicación (prensa, radio, televisión, Internet) para, a través de estos, hacerla llegar a la sociedad.

Pero esto ya no es así. Mejor dicho, esto ya no es solo así. La aparición de la web 2.0 y de las redes sociales ha anulado este estrecho marco de trabajo y ha provocado el surgimiento de un nuevo ecosistema informativo en el que encontramos nuevos elementos, procesos y formas de relación:

I. El periodista, el centro. En el escenario digital de la información cobra protagonismo el periodista frente al medio de comunicación. Si hasta ahora los destinatarios de la información facilitada por el área de comunicación eran los medios, dentro de ellos determinadas secciones y, en último término, los periodistas responsables o especialistas en los distintos temas, ahora el gabinete de prensa debe tener al redactor como punto de partida de una red de difusión en la que ocupa el centro indiscutible. El periodista ha desplazado al medio como referencia del gabinete de prensa. 
La generalización del uso de la web 2.0 por la prensa nos presenta a unos periodistas que trabajan para unas cabeceras pero que simultáneamente escriben y publican en blogs y redes sociales -corporativas y personales- como Facebook o Twitter. Plataformas desde las que difunden el trabajo que han realizado para su periódico, revista, emisora de radio o televisión, pero en las que, además, vuelcan datos y noticias que se han quedado fuera por falta de espacio, tiempo o por criterio de sus superiores. Un nuevo escenario en el que la exclusividad es cada vez más inusual. La colaboración de periodistas en distintos medios, aunque sean competencia directa y pertenezcan a grupos mediáticos distintos, es hoy una práctica común sobre todo en determinadas áreas de información, entre las que destaca la política. Esta red de información tejida por el nuevo periodista en torno a su actividad profesional se amplía también en cuanto a los temas. La web social permite a los redactores escribir y opinar sobre cuestiones que, en principio, están fuera de su sección. Es más, difunden (comparten, retuitean) y opinan (comentan) noticias publicadas por otros colegas en otros medios. El periodista no encuentra más frontera hoy para hablar de un tema que su propio interés.

II. La prensa ya no está sola. Pero la sociedad ya no se informa únicamente a través de lo que cuentan los medios de comunicación tradicionales. Periodistas que se han quedado fuera de las empresas periodísticas, como consecuencia de las reducciones de plantilla y de cabeceras, y profesionales de las más variadas disciplinas o simples ciudadanos, han creado sus propias páginas web o bitácoras y son seguidos por miles de ciudadanos que encuentran en estos sitios informaciones, análisis y detalles complementarios o nuevos sobre los más variopintos asuntos de la vida.

Estos nuevos profesionales de la información deben ser incorporados a las bases de datos de los gabinetes de comunicación y deben ser considerados a todos los efectos como destinatarios habituales de las noticias generadas por dichos gabinetes puesto que participan, como un elemento más, en la cadena de creación, análisis y divulgación de la información. Ya no es extraño encontrar, por ejemplo, a blogueros que han sido captados por periódicos para mantener colaboraciones habituales en la edición impresa, secciones diarias de análisis en la edición digital -a través de blogs creados ex profeso-y para los que se cuenta incluso a la hora de valorar acontecimientos que se están retransmitiendo en directo a través de las redes sociales, como si de un miembro más de la redacción se tratara. Muchos de estos sitios tienen audiencias que superan ampliamente las de algunos medios tradicionales.

III. Apertura del gabinete de prensa. Al igual que han hecho los medios de comunicación tradicionales abriéndose a la colaboración y participación de otros agentes de la información -blogueros, expertos, ciudadanos-, logrando así enriquecer la información de un hecho puntual o con continuidad en el tiempo, los gabinetes de prensa deberán proceder a una apertura de sus estructuras informativas, con carácter esporádico o permanentemente, dependiendo de los 
objetivos de cada organización. La información fluye hoy en todas direcciones y el gabinete de prensa no puede ser un muro de contención sino más bien al contrario, debe aprovechar el paso de los datos y noticias por su área para enriquecer contenidos e impulsar el discurrir de la misma.

Un acontecimiento noticioso que es hoy informado en exclusiva por un gabinete de prensa resulta aburrido. ¿Por qué no facilitar que otros actores relaten, simultáneamente, lo que está pasando y aporten sus puntos de vista? El resultado es, de cara a los periodistas y medios de comunicación a los que nos interesa transmitir lo que está pasando, más información, desde luego, pero sobre todo más transparencia y, por ende, mayor credibilidad. El gabinete de prensa en este nuevo ecosistema informativo surgido en la era digital deberá ser el impulsor de la actividad informativa de la organización, no el que la centraliza para monopolizarla.

IV. Diferentes niveles de profundización. Hasta ahora los diferentes niveles de profundización en la información venían asociados, en primer lugar, a la inmediatez del medio de comunicación. El flash de urgencia lo daba la agencia de noticias y la radio, la televisión esperaba a contar con alguna imagen para hacerlo y el periódico tenía más tiempo para interpretar y analizar lo que había ocurrido. Lo digital ha revolucionado también los tiempos y procesos con los que trabajan los medios de comunicación. Las redacciones funcionan ya con unos esquemas de trabajo, más allá de las características distintivas del medio, que se suceden en diferentes tiempos y con distintos niveles de profundización en la información. En primer lugar, los periodistas que cubren un hecho informativo, sea de agencia, periódico, radio, televisión o sitio de Internet, retransmiten en titulares lo más destacado, a través de Twitter, Facebook o la red social propia del medio. Posteriormente la cabecera ofrece una información más amplia elaborada por el mismo redactor o por otro u otros. Finalmente se profundiza en la noticia con análisis, valoraciones, entrevistas, reacciones de afectados y opiniones de los lectores.

Lejos han quedado los días en los que se convocaba una rueda de prensa o un acto de interés informativo y solo después de realizarla se narraba lo ocurrido, acabando ahí el círculo que debía supervisar el gabinete de prensa, en la primera fase de lanzamiento de una noticia. Lo digital deja a la luz todo el proceso informativo. Los ciudadanos conocen la noticia pero también su gestación. Ello obliga a los responsables de los gabinetes de comunicación a prever con antelación las necesidades de los informadores, facilitarlas y, en su caso, ofrecerlas. Los gabinetes de prensa deberán acostumbrarse a proporcionar a su cada vez más extensa red de seguidores la información también con distintos niveles de profundización: titulares, noticias y entrevistas y análisis, fotos y vídeos. Porque no hay que olvidar que desde las áreas de comunicación de las organizaciones no solo se informa a los periodistas, blogueros, medios tradicionales y nuevos, sino también a los propios de la institución: páginas web y redes sociales corporativas. 
V. En cualquier momento. Hace unos meses el diario El País anunció que integraba sus redacciones en una sola. Los redactores de este diario ya no trabajan para la edición impresa o la edición digital, sino para una sola marca que decide en cada caso, qué va a una y qué a la otra. Uno de sus directores adjuntos en aquel momento, Gumersindo Lafuente, daba más pistas hace unos días y explicaba que el periódico tiene una redacción activa las veinticuatro horas del día, distribuida en tres turnos de trabajo, de tal modo que está continuamente recogiendo, elaborando y actualizando la información que ofrece a sus lectores.

Este ejemplo avanza otra característica de lo digital, que afecta a los gabinetes de prensa y de comunicación. La inexistencia del tiempo y del espacio en Internet implica una disponibilidad permanente de las oficinas de información de las organizaciones, lo que hoy permiten cómodamente las terminales tecnológicas que usamos en nuestra vida diaria. Ya no es de recibo que un medio de comunicación no pueda localizar a un responsable de comunicación de una empresa en fin de semana, si es preciso. Porque, además, y según en qué circunstancia, el no hacerlo puede ser letal para la misma.

VI. Más periodismo. La imagen de los gabinetes de prensa se ha devaluado en los últimos años notablemente. El crecimiento de estas estructuras informativas en las instituciones, organizaciones y empresas ha generado un incremento exponencial de la información que cada día llega a las redacciones de los diarios pretendiendo encontrar un hueco en las noticias que los medios sirven a sus lectores, oyentes o televidentes. Según algunos estudios, los periodistas desechan el $85 \%$ de las notas de prensa que llegan a las redacciones, porcentaje que puede verse aumentado si los gabinetes de prensa no cambian los mensajes que hacen llegar a las redacciones. «Hay notas de prensa que merecen ser destruidas al instante», tuiteaba hace pocos días la periodista de Cinco Días Paz Álvarez.

La comunicación de las organizaciones debe acercarse cada día más al periodismo e irse alejando de la publicidad y el marketing disfrazados de información, habituales e inapropiados compañeros de viaje en los últimos años. No es que las empresas no deban realizar estas actividades, es que no deben mezclarse en las relaciones informativas estrictamente dichas. Los profesionales de los gabinetes de prensa deben esmerarse en generar piezas periodísticas acabadas, que puedan ver la luz en cualquiera de los sitios que habitan la Red y convertirse así en proveedores de contenidos finales de los medios, páginas web, blogs y redes sociales. Pero para ello es preciso que estos contenidos sean creados bajo los estrictos requisitos de la información periodística. Solo la calidad de los contenidos hará posible que una empresa sea visible en el mundo de la información digital.

VII. De tú a tú. En este nuevo ecosistema de la información digital desaparece parte de la burocracia interna de las redacciones de los medios y se establece una relación más directa entre los profesionales de los gabinetes de comunicación y los periodistas. Eso provoca que, partiendo del distinto nivel de profun- 
dización de la información a las que nos referíamos anteriormente, un redactor pueda retuitear inmediatamente una noticia facilitada por la organización, aunque para los niveles siguientes deba ya someterse a la jerarquía del medio. De nuevo la confianza y la credibilidad es un factor imprescindible para que estas situaciones sean posibles.

Las redes sociales establecen conversaciones de tú a tú. Directores de grandes periódicos que difunden comentarios de ciudadanos que les siguen en Twitter, periodistas que comparten noticias de colegas de la competencia, redactores de distintos medios que dialogan o se piden opinión a través de Facebook, es decir, públicamente, sobre acontecimientos que están ocurriendo en distintos lugares del mundo, jefes de sección que retuitean mensajes profesionales o personales de jefes de prensa.

Realmente, este nuevo ecosistema de la información digital es más democrático y rico. Está naciendo. Pero ya se adivina que en él se precisarán unos gabinetes de prensa más comprometidos con el tratamiento periodístico de la información, capaces de asumir parte del trabajo que hasta ahora venían haciendo los medios de comunicación, que deberán asumir nuevas tareas.

\section{Referencia de este artículo:}

Bustos, Rosa de (2012). El gabinete de prensa ante el nuevo ecosistema informativo digital. En: adComunica. Revista Científica de Estrategias, Tendencias e Innovación en Comunicación, $\mathrm{n}^{\circ} 4$. Castellón: Asociación para el Desarrollo de la Comunicación adComunica, Universidad Complutense de Madrid y Universitat Jaume I, 223-227. DOI: http://dx.doi.org/10.6035/2174-0992.2012.4.14 